\title{
Environment status of blue compact galaxies and trigger of star formation
}

\author{
S. A. Pustilnik ${ }^{1,2}$, A. Y. Kniazev ${ }^{1,2}$, V. A. Lipovetsky ${ }^{1, \star}$, and A. V. Ugryumov ${ }^{1,2}$ \\ 1 Special Astrophysical Observatory RAS, Nizhnij Arkhyz, 369167, Karachai-Circassia, Russia \\ 2 Isaac Newton Institute of Chile, SAO Branch
}

Received 26 December 2000 / Accepted 26 March 2001

\begin{abstract}
The work studies ${ }^{\star \star}$ of the environment of low-mass galaxies with active star formation (SF) and a possible trigger of SF bursts due to gravitational interaction. Following the study by Taylor et al. (1995), we extend the search for possible disturbing galaxies of various masses to a much larger sample of 86 BCGs from the sky region of the Second Byurakan survey (SBS). The BCG magnitudes and radial velocities are revised and up-dated. The sample under study is separated by the criteria: $E W\left([\mathrm{OIII}]_{\lambda 5007}\right)>45 \AA$ and $V_{\mathrm{h}}<6000 \mathrm{~km} \mathrm{~s}^{-1}$ and should be representative of all low-mass galaxies which experience SF bursts. We argue that the moderate tidal disturbers should be taken into account, and incorporate the respective range of distances in the search for disturbing neighbours. The majority of the neighbours in the vicinity of the studied BCGs are found through the study of their environment among UZC (Falco et al. 1999) galaxies, and the follow-up careful search of the fainter galaxies in the NED database. For the remaining BCGs, the neighbouring galaxies are found based on the results of the SAO $6 \mathrm{~m}$ telescope spectroscopy. By studing the data on the radial velocities of galaxies in the vicinity of BCGs we found: 1) 33 of the studied BCGs $(\sim 38.5 \%)$ are associated with significantly brighter galaxies $(\Delta B \geq$ $\left.1.5^{\mathrm{m}}\right)$; 2) 23 BCGs ( 26.5\%) have neighbours either of comparable or significantly lower brightness; 3$) 14$ of the studied BCGs (16\%) with no evident associated galaxy are either certain, or probable, mergers. Summarizing, we conclude that in $\sim 80 \%$ (or more) BCGs from the studied sample, the SF bursts are triggered either by tidal action of various strengths from other galaxies, or due to mergers of low-mass galaxies. We briefly discuss the implications of our main conclusion for evolutionary links of BCGs to other types of low-mass galaxies. Part of our sample falls into the volume belonging to the Local Supercluster. Therefore we formulate the results separately on the "Local Supercluster volume" and "general field region". The total fractions of BCGs likely triggered by interaction with other galaxy are respectively, $\sim 84.5 \%$ and $80 \%$ for the nearby volume and for the general field. The fractions of BCGs with significantly brighter disturbers in these two groups are seemingly different $(\sim 54 \pm 14 \%$ vs. $\sim 31.5 \pm 7 \%$, respectively). Among the so called "isolated" BCGs (that is, without a bright neighbouring galaxy) in both the Local Supercluster volume and in general field, $\sim 43 \pm 10 \%$ are probably disturbed by dwarf galaxies and $\sim 26 \pm 8 \%$ have a merger morphology. In the Appendix we present the results of the spectroscopy with the SAO $6 \mathrm{~m}$ telescope of 27 galaxies in an attempt to find possible disturbing galaxies in the vicinity of some of the sample BCGs.
\end{abstract}

Key words. galaxies: dwarf - galaxies: interaction - galaxies: evolution - galaxies: starburst

\section{Introduction}

Blue compact galaxies (BCGs) with strong emission lines or HII-galaxies are low-mass objects with the current star-formation (SF) rate exceeding many times this parameter averaged over cosmological time. The observed $\mathrm{SF}$ rates for most BCGs are so high that star formation could be sustained at the current level only on a timescale

Send offprint requests to: S. Pustilnik, e-mail: sap@sao.ru

* Deceased 1996 September 22.

** Tables 2, 3 and A.1 with their notes and Figs. A.1-A.3 are only available in electronic form at http://www . edpsciences .org significantly lower than $1 / H_{0}{ }^{1}$ before their neutral gas reservoir will be completely depleted (e.g., Thuan 1991). This implies that either these objects are rather young, with typical ages of less than one or a few Gyr, or SF in BCGs is highly variable and proceeds in short episodes (bursts) with a typical duration of 10-100 Myr, intermittent with long periods of SF activity at levels much lower than that during the SF burst. In the course of such SF bursts, BCGs experience significant brightening relative to their quiescent state - an average of 0.75 in the small BCG sample from Papaderos et al. (1996), and

${ }^{1} H_{0}$ is the Hubble constant, accepted hereafter as $75 \mathrm{~km} \mathrm{~s}^{-1} \mathrm{Mpc}^{-1}$. 
up-to $\sim 2{ }^{\mathrm{m}} 0$ in a large sample from Lipovetsky et al. (2001). Therefore being in general low-mass and underluminous systems with a wide range of baryon (stars+gas) mass $\left(10^{7}<M_{\text {bar }}<10^{10} M_{\odot}\right)$, BCGs sometimes can be as bright as $M_{B}=-20{ }^{\mathrm{m}} 0$.

In the great majority of properly studied BCGs, the traces of old low-mass populations are detected (e.g., Thuan 1983). This implies that, in general, BCGs are rather old objects, and their evolution on the Hubble time scale can effectively be determined by short, but very intensive SF, bursts. The option of truly young galaxies is still probable for very small fraction of BCGs $(\sim 1 \%)$ with extremely low metallicities (e.g., Izotov \& Thuan 1999; Kniazev et al. 2000; Pustilnik et al. 2001a).

While the mechanisms that regulate and terminate SF bursts on the "short" time scales are more or less known and partly understood (SNe and stellar winds), the nature of the mechanisms which trigger the collapse of gas clouds and the onset of SF bursts in low-mass galaxies has been debated since the early 70 -s.

A general approach to the question should take into account that even though BCGs do not comprise a homogeneous class of objects (since they possess a wide range of observed morphologies), to a first approximation they all are gas-rich galaxies with high enough specific angular momentum, and therefore more or less flattened rotating "disks" (e.g. Taylor et al. 1993, 1995; Chengalur et al. 1995; van Zee et al. 1998). In the least massive BCGs, the rotation energy is not much greater than that of random motion, and their "disks" are thicker. On the other hand the BCGs similar to Mkn 996 that is, with the light distribution typical of elliptical galaxies are quite rare (Doublier et al. 1997). The latter is probably indicative of their post-merger nature (Thuan et al. 1996).

The concept of equilibrium and gravitational instability in a rotating disk is based on the local stability criterion, introduced by Toomre (1964). There is a threshold surface density (depending on the local epicyclic frequency and the dispersion of the random velocity component, Toomre 1964; Kennicutt 1989), above which gas is unstable. Positions of SF regions in disk galaxies, examined on a variety of galaxy types (Sa-Sm, Im, LSB) are consistent with the criterion of Toomre-Kennicutt (Kennicutt 1989; van der Hulst et al. 1993). Positions of SF regions in BCG/H II galaxies also fulfill this criterion (e.g., Taylor et al. 1994; van Zee et al. 1998).

On the other hand, most of the observed cases of enhanced SF in massive gas-rich galaxies are associated with various interactions from other galaxies. Keel (1993), from the kinematical study of a well-selected sample of interacting galaxies has drawn the important conclusion that gravitational instability in their disks is driven initially by external perturbations (see also Bernloehr 1993).

At a first glance this mechanism could play an important role for BCGs as well. However, the studies of the spatial distribution of BCGs indicate that only a minority are tightly connected to massive galaxies or their systems (e.g., Salzer 1989; Campos-Aguilar \& Moles 1991; Vilchez 1993; Pustilnik et al. 1995).

This fact does not imply that interactions are of low significance as SF triggers in BCGs. However many researchers seem to favor some internal process as the main trigger of enhanced SF. In particular, the mechanism suggested by Gerola et al. (1980), which is based on the stochastic collisions and merging of large gas clouds is noted. Another type of internal trigger is connected with the hypothesis of cyclic gas re-processing, with the characteristic time between the subsequent SF bursts related to the fall-off back to the "disk" of the gas lifted to the halo during the previous SF burst (Salzer \& Norton 1999).

The two alternative models have been suggested to overcome the apparent difficulties of the hypothesis in explaining the interaction-induced SF burst in BCGs, as evidenced by their weak spatial connection to massive galaxies. The first, mentioned by Melnick (1987) and clearly formulated by Brinks (1990), incorporates the tidal action from nearby low-mass galaxies. It was tested in the VLA search for HI-rich companions of 19 nearby $(V<$ $2500 \mathrm{~km} \mathrm{~s}^{-1}$ ) "isolated" H II-galaxies (Taylor et al. 1995; see also Taylor 1997). Surprising, 10 of the 19 target galaxies revealed low-mass HI companions, most of which were identified later with faint galaxies. However, the statistics are still rather sparse. Some additional indications of the potentially important role of low-mass galaxies appeared after the deep CCD imaging of the large BCG sample by Lipovetsky et al. (2001).

Another model of an external trigger for SF bursts in BCGs was suggested by Östlin et al. (1999) and Kunth \& Östlin (2000). Based on morphology and gas kinematics data for the small sample of luminous BCGs, they consider that mergers of low-mass galaxies are the most important factor affecting the group of BCGs. Similar results were reported by Sung (2000), based on studies of a sample of 115 BCG/H II-galaxies.

Thus, during the last 5-7 years, an increasing amount of evidence has appeared which imply the importance of gravitational interaction of low-mass galaxies with current SF bursts, in particular with galaxies of comparable or lower mass. At the same time, neither new simulation has demonstrated the potential of an internal spontaneous onset of SF bursts in gas-rich galaxies, nor do any concrete models indicate the physical conditions which are necessary for this mechanism to apply.

Therefore the relative role of external and internal trigger mechanisms of SF in BCG/H II-galaxies seems far from to being settled. It is high time to pose the question: what are the main trigger mechanisms of SF burst in BCGs? This is important both for the general understanding of the BCG phenomenon and the cosmological evolution of BCGs.

In this paper, we use the well-selected BCG sample of 86 galaxies in the zone of the Second Byurakan Survey to get the quantitative estimate of the relative frequency of gravitational interaction as a probable trigger of SF bursts in BCG progenitors. Based mainly on UZC 
(Falco et al. 1999) and the $\mathrm{NED}^{2}$, we perform an extensive search for nearby disturbing galaxies, both more massive and those of comparable or lower luminosity than that of the studied BCGs. For BCGs without such probable disturbers in UZC and NED and in recent publications on galaxy redshifts, we have measured the radial velocities of some candidate disturbers with the SAO $6 \mathrm{~m}$ telescope.

The paper is organized as follows. In Sect. 2 we briefly review tidal trigger of $\mathrm{SF}$, discuss in more detail the most efficient examples, and describe the criteria we apply to assign a nearby galaxy as a disturbing neighbour. In Sect. 3 we describe our BCG sample, while in Sect. 4 we present the results of the "companion" search and explain the sources we have used. A brief analysis of the properties of galaxies, associated with BCGs, the discussion of results obtained and our conclusions are presented in Sect. 5. The results of spectroscopy of 3 BCGs and 24 candidate companions in the vicinity of 15 studied BCGs are presented in the Appendix.

\section{Tidal trigger}

Enhanced SF activity in large gas-rich galaxies is stimulated by various types of galaxy interactions, including: a) mergers of close mass galaxies (e.g., Larson \& Tinsley 1978; Joseph et al. 1984); b) sinking dwarf companions (e.g., Mihos \& Hernquist 1994; Rudnick et al. 2000); c) strong tidals (e.g., Bernloehr 1993; Keel 1993); d) weak tidals (e.g., Icke 1985; Reshetnikov \& Combes 1997; Rudnick et al. 2000). In principle, all these mechanisms could also work for gas-rich low-mass galaxies. The relative importance of these external triggers, as well as their role in comparison to some internal triggers, seemingly should depend on the type of environment.

While a detailed understanding of trigger mechanisms of enhanced SF due to the tidal action of a nearby galaxy on gas-rich dwarfs is still lacking, several schemes were suggested to model the effect of galaxy interaction, which can be applied to low-mass galaxies. The method of Noguchi (1988) is based on the generation of a central bar which in turn disturbs gas clouds and causes them to sink towards the center of galaxy, inducing a central SF burst. Another mechanism, suggested by Olson \& Kwan (1990), works via a large tidal increase in the inelastic collision rate of individual gas clouds, and their merging and collapse. It is basically a stochastic process. Both these mechanisms require strong enough tidal disturbance, possible when the fly-by of equal mass galaxies takes place at the pericenter distance of about 8-10 disk scale-lengths.

The mechanism suggested by Icke (1985) involves the generation of shocks in the outer parts of the HI disk, with the subsequent dissipation of their kinetic energy and the loss of dynamical stability of the gas disk. It takes a moderate tidal force, and under similar conditions effectively acts from a distance $2-3$ times larger than the two former

\footnotetext{
2 http://nedwww.ipac.caltech.edu/ - the NASA-IPAC Extragalactic Database.
}

models. Also, the cross-section of the Icke mechanism is several times larger.

This circumstance favors the Icke mechanism as the most efficient in the stimulation of abnormal SF activity. Some indirect arguments to support the importance of this trigger mechanism are the traces of recent enhanced SF in spiral galaxies with lopsidedness and bent outer parts. The latter are considered to be evidence of minor tidal effects (e.g. Rudnick et al. 2000; Reshetnikov \& Combes 1997). However, lopsidedness can be caused by minor mergers as well (Rudnick et al. 2000). Evidently, the two former, or similar, mechanisms also can work to trigger BCG progenitors. However, their role is probably less important.

To illustrate quantitavely the parameters of the Icke mechanism and the pericenter distance at which tidals become effective at generating shocks, we give below the simple formulae taken from the paper by Icke (1985).

The Icke mechanism is based on tidal acceleration of gas layers to supersonic velocities and the subsequent dissipation of shock waves. A characteristic value for the speed gained by gas due to the tidal acceleration by an "intruder" (the object which exerts the tidal force; the name does not necessarily imply a close approach) over a time span corresponding to one revolution (a characteristic time in the galaxy-"victim" system) is given by the formula:

$$
\delta v \approx 4 G M R \cdot r^{-3} \cdot 2 \pi R / v=8 \pi \cdot v(R / r)^{3},
$$

where $M$ is the mass of the galaxies, assumed here to be equal, $R$ - is the radius of the external part of the galaxyvictim disturbed by the tidal interaction, $r$ is the distance between the galaxy centers, and $v$ is the circular speed at the radius $R$, assumed to be equal to $v=(G M / R)^{1 / 2}$. From the condition that the shock can occur, that is, $\delta v>$ $s_{0}\left(s_{0}\right.$ is "sound" speed), one gets the condition for the pericenter distance $p_{0}$, at which the tidal interaction will lead to shock generation: $p_{0} \approx R \cdot\left(8 \pi \cdot v / s_{0}\right)^{1 / 3}$.

For the general case of colliding galaxies of unequal masses, with the mass ratio $\mu=M$ (intruder) $/ M$ (victim) the latter relation changes to: $p_{0} \approx R \cdot\left(8 \pi \cdot \mu v / s_{0}\right)^{1 / 3}$.

To illustrate the range of the expected values of $p_{0}$ for several representative cases of the mass ratio, we calculate this according to the formulae above for two low-mass "disk" galaxies with typical of BCG parameters. We accept here $s_{0}=10 \mathrm{~km} \mathrm{~s}^{-1}$ as the characteristic velocity dispersion in the interstellar gas. One case corresponds to a galaxy with $M$ (victim) tot $=10^{10} M_{\odot} ; v / s_{0}=7$; $R($ victim $)=10 \mathrm{kpc}$ and another - to a galaxy with $M(\text { victim })_{\text {tot }}=10^{9} M_{\odot} ; v / s_{0}=3 ; R($ victim $)=5 \mathrm{kpc}$. $R$ (victim) is taken here as the size of the outer HIdisk, corresponding to the characteristic mass density of $\sim 0.5 M_{\odot} / \mathrm{kpc}^{2}$. We notice that according to observations, BCGs, as well as other dwarfs, are dominated dynamically by Dark Matter halos, and their baryonic mass is several times lower than their total mass. So a DM halo plays the same role as a spherical stellar halo in the original Icke model. The respective values of $p_{0}$ for $\mu=200,10,1$ and 
0.1 are shown in Table 1 . The parameters above correspond to the typical ones of BCGs, as described, e.g., by Taylor et al. (1995). According to the same authors, BCGs with such total masses can have a wide range of blue luminosities, corresponding to $M_{B}=-12.7$ to -17.0 .

Table 1. Characteristic threshold pericenter distance

\begin{tabular}{rrrrrr}
\hline$M$ (vict) tot & \multicolumn{1}{c}{$10^{10} M_{\odot}$} & & \multicolumn{2}{c}{$10^{9} M_{\odot}$} \\
\hline$M($ intrud $)$ & $\mu$ & $p_{0}$ & $M($ intrud $)$ & $\mu$ & $p_{0}$ \\
$\left(\mathrm{M}_{\odot}\right)$ & & $(\mathrm{kpc})$ & $\left(M_{\odot}\right)$ & & $(\mathrm{kpc})$ \\
\hline $2 \times 10^{12}$ & 200.0 & 327 & $2 \times 10^{11}$ & 200.0 & 123 \\
$10^{11}$ & 10.0 & 121 & $10^{10}$ & 10.0 & 45 \\
$10^{10}$ & 1.0 & 56 & $10^{9}$ & 1.0 & 21 \\
$10^{9}$ & 0.1 & 26 & $10^{8}$ & 0.1 & 10 \\
\hline
\end{tabular}

One important note related to the problem of the observational search for potential "intruders" around the target BCGs is connected to the expected time delay between the passage of the pericenter and the beginning of SF burst. For example, for $V_{\text {rot }}=70 \mathrm{~km} \mathrm{~s}^{-1}$ and $R=10 \mathrm{kpc}$, the characteristic time of development of shocks is comparable (Icke 1985 ) to $T=2 \pi R / V_{\text {rot }}=8 \times 10^{8} \mathrm{yr}$. In general, checking any nearby galaxy as a potential "intruder" of the target BCG, we need to account for the relative tangential velocities of the two considered galaxies $\delta V$, in our case up to $300-400 \mathrm{~km} \mathrm{~s}^{-1}$. Thus, e.g., for $\delta t=T / 2$ for the disk above, and $\delta V=200 \mathrm{~km} \mathrm{~s}^{-1}$, by the time of a well-developed SF episode, the two galaxies can increase their projected separation from their pericenter position by $90 \mathrm{kpc}$. Hence, due to the time delay between the maximum tidal action and its consequence as a SF burst, one can expect that even relatively low-mass neighbours can be the real disturbing galaxies if they are found at distances of less than $\sim 100-150 \mathrm{kpc}$. The delays in the beginning of a SF burst in the more massive component in pairs of interacting spirals with highly different masses were noticed by Bernloehr (1993). These delays range up to several hundred Myr, which in general is compatible with the numbers given above.

Sufficiently detailed observational data for BCG companions to be used to test the role of relatively weak tidals are not abundant. The most suitable are seemingly the results of the search for low-mass HI companions near H IIgalaxies by Taylor et al. $(1993,1995)$. The projected distances in the systems with detected HI companions are consistent with the estimates of the threshold pericenter distances with input observational parameters, if again, some reasonable delay in the ignition of SF bursts is taken into account.

We also note that to directly detect shocks in HI gas of BCGs triggered by an interaction is not simple. According to the model, they dissipate to initiate gas instability. Therefore, by the time of the developed SF burst, the signs of shocks can be completely erased. Therefore the search for such disturbed velocity fields should be directed to the systems in the earlier stages of collision, in which an SF burst has not yet occured. Probably gas-rich galaxy pairs with a large mass difference, such as those studied by Bernloehr (1993), can be suitable for this task.

\section{BCG sample}

The BCG sample for this study is compiled from the BCG sample in the zone of the Second Byurakan Survey $(\mathrm{SBS})\left(\mathrm{RA}=7^{\mathrm{h}} 40^{\mathrm{m}}-17^{\mathrm{h}} 20^{\mathrm{m}}\right.$, Dec $\left.=49^{\circ}-61^{\circ}\right)$. The latter is partly described by Izotov et al. (1993), Thuan et al. (1994), Pustilnik et al. (1995). The same BCG sample was in particular the subject of an HI-study by Thuan et al. (1999). Their subsample of 88 BCGs is reduced slightly here to match the strict criterion of the strength of the [OIII]-line.

Our sample includes 86 BCGs with a sufficiently strong [OIII]-line $\left(E W\left([\mathrm{OIII}]_{\lambda 5007}\right)>45 \AA\right)$ and $V_{\text {hel }}<$ $6000 \mathrm{~km} \mathrm{~s}^{-1}$. The first criterion corresponds to the line strength at which the loss of candidate emission-line galaxies (ELGs) from objective prism plates during the primary selection is moderate, while the second criterion is to limit the volume where search for non-massive neighbours can be efficiently carried out. While the question of completeness analysis of SBS BCG sample is beyond the scope of this paper, it is useful to describe the bias inherent to the BCG sample. As demonstrated by Salzer (1989), the selection in emission-line surveys is determined by the total flux in line+continuum. Thus, for apparent magnitudes close to the threshold of photographic plates, only emission lines with large enough equivalent widths $(E W \mathrm{~s})$ will be detected. From the carrying out of such selection on the SBS plates and the preliminary comparison in the small overlapping region of SBS detected BCGs with BCGs detected in the Hamburg/SAO survey (Ugryumov et al. 1999), for the limiting $m_{B}=18$, which is near the completness limit of the SBS sample (Pustilnik et al. 1995), galaxies having HII-regions with $E W\left([\mathrm{OIII}]_{\lambda 5007}\right)>(45-50) \AA$ are selected with modest loss, and thus can be considered as representative of the whole population of galaxies with that level of SF activity. This level corresponds, for typical extragalactic H II-regions, to $E W(\mathrm{H} \beta)>(10-20) \AA$, which in turn in the model of instantaneous SF burst corresponds to the burst age $<(7-10)$ Myr (Leitherer et al. 1999). Therefore, the main bias introduced by the applied criteria to the entire BCG/HII-galaxy population is the selection of relatively young SF bursts, which can more easily be found in objective prism spectral plates. Some characteristics of the sample are: the range of apparent blue magnitudes $B=12.5 \div 18.5$, the range of absolute blue magnitudes $M_{B}=-11^{\mathrm{m}} 0 \div-19 \mathrm{~m}$. For many of these galaxies, the accuracy of radial velocities was significantly improved after HI-line measurments by Thuan et al. (1999) and additional optical spectroscopy (Izotov et al. 1994; Thuan et al. 1995). To illustrate the properties of this BCG sample, we show in Fig. 1 some of its parameter distributions $\left(m_{B}, M_{B}, V_{\text {hel }}\right)$. 

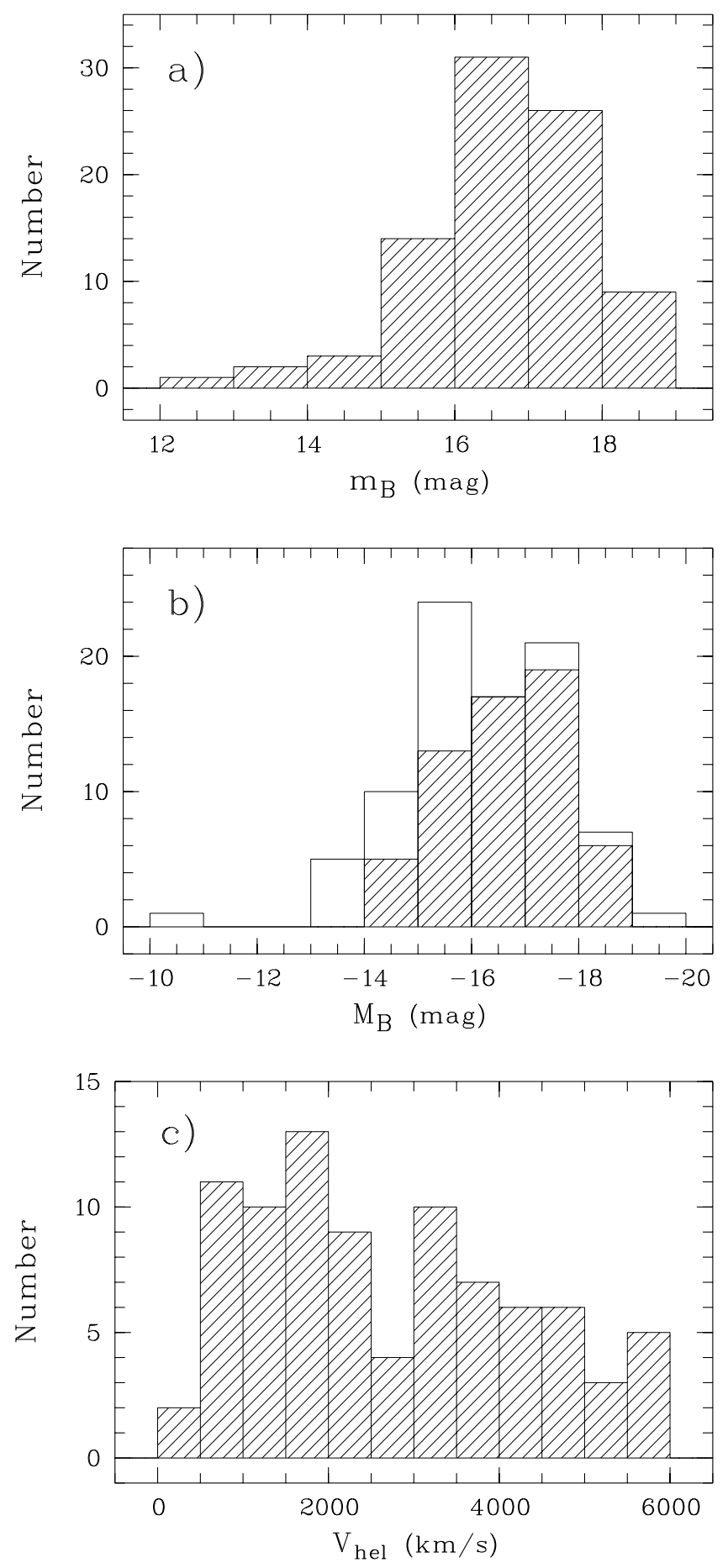

Fig. 1. Distributions of the BCG sample on $m_{B}$ a), $M_{B}$ b) and $\left.V_{\text {hel }} \mathbf{c}\right)$. The hatched and unhatched parts of the $M_{B^{-}}$ distribution separate BCGs from the general field group (60 galaxies) and the Local Supercluster volume (26 galaxies) respectively. See Sect. 5.3 for details.

One can see that the sample is more or less typical, with some excess of the less luminous BCGs in comparison to e.g., the BCG sample in the same sky region studied by Pustilnik et al. (1995). This is connected to the different velocity boundaries adopted for the latter paper $\left(2000 \mathrm{~km} \mathrm{~s}^{-1}<V_{\text {hel }}<10000 \mathrm{~km} \mathrm{~s}^{-1}\right)$ and ours
$\left(V_{\text {hel }}<6000 \mathrm{~km} \mathrm{~s}^{-1}\right)$. This sample includes a significant number of BCGs located inside the Local Supercluster that could bias the conclusions relative to the situation in the general field. This question will be addressed in the discussion section. B-magnitudes of many BCGs in the considered sample are significantly corrected based on the results of CCD-photometry (Lipovetsky et al. 2001; Kniazev et al. 2001a).

The list of the studied sample of 86 BCGs is presented in Table 2, containing the following information:

Column 1: The object's IAU-type name. For those without an alternative name the prefix SBS is applied in the last column.

Column 2: Right ascension (RA) for equinox B1950. The coordinates of BCGs in this table sometimes differ from those presented in NED, but are the most up-to-date, according to our own checks on the Digitized Sky Survey (DSS).

Column 3: Declination for equinox B1950.

Column 4: Heliocentric velocity in $\mathrm{km} \mathrm{s}^{-1}$. For more than 60 BCGs the r.m.s. uncertainty is less than $30 \mathrm{~km} \mathrm{~s}^{-1}$ (mainly from HI data). For seven BCGs it can reach $\sim 100 \mathrm{~km} \mathrm{~s}^{-1}$, and for the remaining galaxies it is $\sim 50$ $60 \mathrm{~km} \mathrm{~s}^{-1}$.

Column 5: Reference to the source of velocity accepted, coded by 1 - Thuan et al. (1999): 2 - NED; 3 - Pustilnik et al. (1995); 4 - new measurments with the SAO $6 \mathrm{~m}$ telescope (this paper, Appendix); 5 - UZC: Falco et al. (1999); 6 - Carrasco et al. (1998). Some of our BCG velocities in NED, taken from HI-measurments by Thuan et al. (1999), are not reliable, since they ignore the information on real or possible confusion. We used instead for these galaxies the velocities from Pustilnik et al. (1995).

Column 6: Distance in Mpc, accounting for Virgo infall correction from Kraan-Korteweg (1986) with $V_{\text {infall }}=$ $220 \mathrm{~km} \mathrm{~s}^{-1}$. For one nearby galaxy $\left(V_{\text {hel }}=289 \mathrm{~km} \mathrm{~s}^{-1}\right)$, marked by an asterisk, the distance is accepted from Georgiev et al. (1997). For another similar galaxy $\left(\mathrm{Mkn} 178, V_{\mathrm{hel}}=249 \mathrm{~km} \mathrm{~s}^{-1}\right)$, the distance is accepted to be equal to that of its neighbours on the sky with close radial velocities and having photometrical determinations. Column 7: Total B-magnitude from the unpublished CCD data (Lipovetsky et al. 2001; Kniazev et al. 2001a). They are marked by a "plus" before the value in the corresponding column. For several galaxies, $B$-magnitudes are based on the APM database values (Irwin 1998). They were recalculated to the standard CCD $B$-magnitudes using the relation between $B_{\mathrm{CCD}}$ and $B_{\mathrm{APM}}$ derived on more than 100 galaxies, as described by Kniazev et al. (2001b). Its rms uncertainty is found to be 0.45 . The $B$-magnitudes obtained in this way are marked by an "asterisk" before the value. For a couple of objects, photometrical $B$ magnitudes are taken from NED. They are marked by a "n" before the value.

Column 8: Absolute B-magnitude, calculated from the apparent magnitude and the distance in Col. 5 , with no correction for the Galaxy extinction, since it is small for considered sky region. 
Column 9: A flag to distinguish the galaxies belonging to the groups of the two types of environment (LS Local Supercluster, GF - general field). See Sect. (5.3) for details.

Column 10: One or more alternative names, according to the information from NED.

\section{Search for neighbours and results}

\subsection{Search for neighbouring galaxies}

We employed the following sequence for the search for neighbours of the sample BCGs. As a first pass we checked possible nearby galaxies in UZC, using the criterion $|\Delta V|<500 \mathrm{~km} \mathrm{~s}^{-1}$ and $d_{\text {proj }}<700 \mathrm{kpc}$. For the second pass, we used NED in order to check whether some fainter galaxies are present in the BCG environment which could exert a stronger tidal action than the candidates found (if any) from UZC. The same criterion was applied as for the first pass, and then the candidates were additionally checked for correspondance of their projected distances to those for potential intruders, illustrated in Table 1. We also used some unpublished results on the redshifts of SBS galaxies, as well as our own results of dedicated spectroscopy of potential candidate companions. Since the galaxies, picked up in NED, present a mixture of the content of many catalogs as well as some small groups found in surveys of varying powers, it is difficult to address the question of completeness for the group of neighbouring galaxies found in the vicinity of BCGs. Therefore we can only emphasize that our estimates of neighbouring galaxies found this way are the lower limits of real numbers.

\subsection{Results and types of neighbours}

The results of the search for probable disturbing/interacting galaxies with the sufficiently strong tidal action on the sample BCGs are summarized in Table 3. We present the candidate disturbing galaxy which exerts the strongest tidal action on the target BCG. If there are other galaxies with comparable effect, we indicate them in the Notes on individual BCGs after the table. Table 3 contains the following data:

Column 1: BCG IAU-type name. The same as in Table 2. Column 2: The name (or one of the best known names, such as NGC one) of the suggested disturbing galaxy. Prefix " $\mathrm{S}$ " in the name means the object is from SBS. Prefix "A" means a new object (anonymous), found as an associated galaxy in the spectroscopy data from the $6 \mathrm{~m}$ telescope. In the case of a BCG supposed to be a merger, its name is repeated in this column. If neither merger morphology nor disturbing galaxy is identified, this field is left blank.

Column 3 and 4: RA and Declination of the disturbing galaxy for the equinox B1950. Not given for a suggested merger, or if no disturbing galaxy is identified.

Column 5: Heliocentric velocity of BCG, in $\mathrm{km} \mathrm{s}^{-1}$. Same as in Table 2 .
Column 6: Heliocentric velocity of the disturbing galaxy, in $\mathrm{km} \mathrm{s}^{-1}$. When the velocity of the disturbing galaxy, originally found in UZC, was known in NED with a better precision, we cite the NED value.

Column \%: The source of the value in the previous column, same coding as in the description for Table 2.

Column 8: B-magnitude of the disturbing galaxy. Most are taken from NED. The rest are either recalculated from the APM $B$-magnitudes through calibration with the CCDbased $B$-magnitudes, similar to those in Table 2, as described by Kniazev et al. (2001b), and marked by "** before the value, or are derived from the same CCD-frames on which target BCGs were measured. These are marked by "+".

Column 9: Absolute B-magnitude of the disturbing galaxy, calculated with the same distance modulus as for the neighbouring BCG.

Column 10: Difference of the absolute magnitudes of BCG and its disturbing galaxy $\Delta M_{B}=M_{B}$ (partner) $M_{B}$ (BCG). A negative value means that the disturbing galaxy is brighter.

Column 11: The projected distance between the BCG and the disturbing galaxy, in kpc.

Column 12: Suggested trigger classification: $\mathrm{p}$ - parent galaxy, when the disturbing galaxy is significantly brighter than the target BCG; b - binary system, in which the target BCG and its disturbing galaxy are dynamically comparable; $\mathrm{f}$ - fainter companion, when the disturbing galaxy is significantly less massive than the target BCG; $m$ and $\mathrm{m}$ ? - merger morphology with the various degrees of confidence. The latter classification was performed through the comparison of the morphology of candidate BCGs on the CCD frames from Lipovetsky et al. (2001) and Kniazev et al. (2001a), or on the DSS-2 images, with the galaxies shown by Keel \& Wu (1995).

From the data in Table 3, one can see that the most representative group of 33 BCGs in this sample $(\sim 38 \pm 7 \%)^{3}$ have close enough brighter galaxies (conditionally classified as parent) with $\Delta M_{B}$ in the range $-1.5^{\mathrm{m}}$ to $-6^{\mathrm{m}}$. 23 BCGs from this sample $(\sim 27 \pm 6 \%)$ are found to have disturbing galaxies of comparable brightness, or even significantly fainter. Of the remaining BCGs, which have no evident "neighbours", 14 BCGs ( 16 $\pm 4 \%)$ show more or less evident merger morphology. Only 16 BCGs $(\sim 18.5 \pm 5 \%)$ have no clear indication of interaction with other galaxy. However, for many of these BCGs there are faint galaxies in their vicinity with still unknown redshifts. So the fraction of "non-interacting" BCGs presented here should be rather treated as an upper limit.

Thus, our results indicate that gravitational interaction between gas-rich BCG progenitors and various mass galaxies in their environment may play a key role in the ignition of a SF burst. In particular, the conclusions by Taylor et al. $(1995,1997)$ on the important role of

\footnotetext{
3 Here and in other presented fractions the errors are given based on Poisson statistics of numbers of BCGs involved in each group.
} 

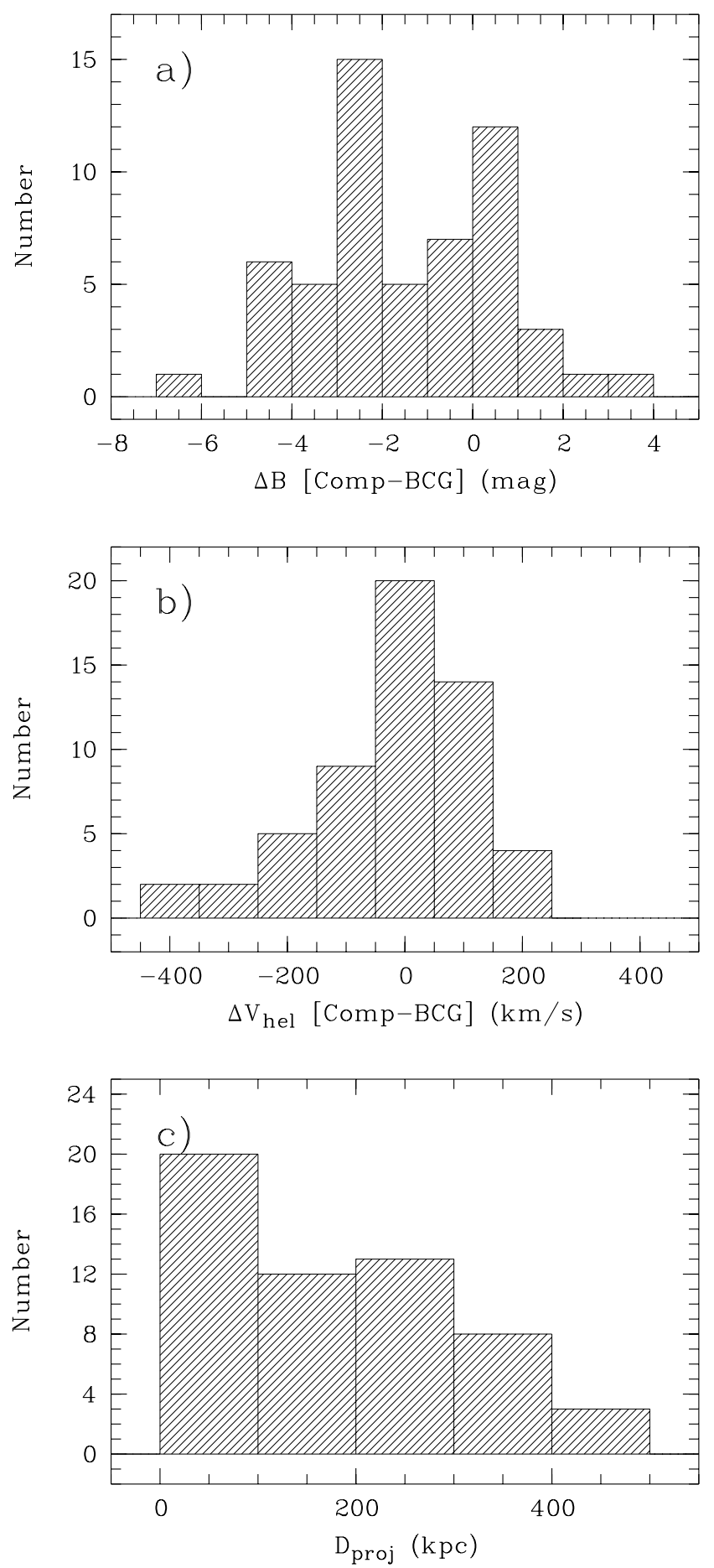

Fig. 2. a) Distribution of blue absolute magnitude diffirences of BCGs and their disturbing galaxies; b) Distribution of the relative radial velocities of BCGs and their disturbing galaxies; c) Distribution of the projected distances between BCGs and their partner galaxies.

low-mass companions in triggering SF in "isolated" H IIgalaxies are consistent with our data. Similar conclusions are drawn by Noeske et al. (2001), based on the environment study of BCGs, mainly from the UM survey. The obtained results do not exclude that some internal mechanisms, which can trigger SF bursts in BCGs, probably work in the minority of them under certain conditions. However, in the majority of BCGs, the external trigger should be more likely, connected to interactions with other galaxies.

\section{Discussion and conclusions}

\subsection{Companion properties}

It is interesting to examine the properties of the found disturbing galaxies and to describe in more detail the relative fractions of various combinations of BCGs with their partner galaxies.

In Fig. 2 the distributions of blue absolute magnitude differences, the relative projected distances and the relative radial velocities are shown.

One can see that the general distribution of the projected distances for suggested disturbing galaxies spreads upto $\sim 400 \mathrm{kpc}$, which is consistent with conclusions by Zaritsky et al. $(1994,1997)$ on the distribution of companions around massive spirals. However, if we limit the group of disturbing galaxies by "dwarfs" $\left(M_{B} \geq-18.5\right)$, then the distribution of the projected distances is much narrower, with the median $D_{\text {proj }} \sim 90 \mathrm{kpc}$. The distribution of the relative radial velocities of the partner galaxy and the BCG peaks at $\Delta V=0$, with $\sim 93 \%$ of this value in the range $\pm 250 \mathrm{~km} \mathrm{~s}^{-1}$. The latter value is typical for these studies, considering possible physical neighbours of field galaxies.

In Fig. 2 the full range of total magnitude differences is shown for BCGs with identified disturbing partners. Its asymmetric (relative to zero) and bimodal appearance at least partly is caused by selection effects. It is more difficult to measure the redshifts for the fainter candidate neighbours. However, even if all remaining BCGs without identified disturbing galaxies would appear to have fainter companions after a more careful search, this distribution will be still bimodal, with the prominent peak at $\Delta B$ around $-2 \cdot 5$. Accounting for the mean brightening of BCGs due to a SF burst of the order $\Delta B=0.75$ 1. 0 (e.g., Papaderos et al. 1996; Lipovetsky et al. 2001), the full interval of the mass ratios of the BCG progenitor and its disturbing galaxy ranges from $\sim 1 / 400$ to $\sim 25$. The peak in the distribution of $\Delta B$ indicates that the most common brighter disturbing galaxies are more massive than the BCG progenitors by a factor of $10-25$.

\subsection{The problem of a control sample}

For confident conclusions on the results of statistical studies like this, it is quite important to compare them to the results of a similar study on a control sample. The objects of the latter should be separated to have a complimentary property relative to that of the main sample. In our case, it should be a sample of gas-rich, low-mass galaxies with no SF bursts. However, while there is no problem in obtaining a large, well-selected BCG sample in the considered volume, it is very difficult to form such a sample of 
LSB dwarfs or Im galaxies. We postpone the similar study of control samples to the time when such samples will be available, hopefully after SDSS (Sloan Digital Sky Survey) data will be distributed. For the purposes of illustration, we undertook the study of the environments for the sample of LSB dwarfs by Pildis et al. (1997). We separated from their 110 LSB dwarfs, 56 objects with $V_{\text {hel }}<6000 \mathrm{~km} \mathrm{~s}^{-1}$ and $V_{\text {Vir }}>2000 \mathrm{~km} \mathrm{~s}^{-1}$, that is, with the same criteria as for our general field (GF) BCG group (see next subsection).

Before comparing this group of LSB galaxies with BCGs for the presence of disturbing neighbours, it is reasonable to check how similary they are distributed in space (that is, in radial velocity), and how similar are their ranges of global parameters, like luminosity and gas mass. In Fig. 3 we show distributions of $V_{\mathrm{r}}, L_{B}$, and HI mass for both samples. We note that $V_{\mathrm{r}}$ and $L_{B}$ distributions for BCGs and LSB galaxies are similar. The null hypothesis on the same distribution of both samples can not be rejected, according to the Kolmogorov-Smirnov $D$ statistic, even on the significance level 0.37 and 0.31 , respectively. This makes the comparison of distances to the disturbing galaxy secure for the two samples.

For $M(\mathrm{HI})$, despite the closeness in the total range of this parameter for both samples, their distributions have larger differences, which is also reflected in the Kolmogorov-Smirnov $D$ statistic (significance level for rejection of the null hypothesis is 0.03 ). This difference, however, is only of the general interest. The amount of HI in the considered galaxy samples can be the result of the difference of their distances to the disturbing neighbours, but can not be the reason for this difference.

To compare the distributions of LSB galaxies and BCGs according to the distance to the most disturbing galaxy, we proceeded the same way as for BCGs, but have checked potential disturbers at the projected distances upto $3 \mathrm{Mpc}$. First, we checked potential neighbours in UZC (Falco et al. 1999), then looked at possible fainter neighbours in NED. Finally, we selected the most tidally disturbing galaxies, assuming mass to be proportional to $L_{B}$, and the tidal force proportional to the cubed inverse projected distance $D^{-3}$.

The search for the strongest disturbing galaxies for this LSB subsample have been made in UZC and NED, taking the limiting difference in radial velocity $|\Delta V| \leq$ $500 \mathrm{~km} \mathrm{~s}^{-1}$. Its results are illustrated in Fig. 4 along with the results of a similar search for BCGs from the GF group. For BCGs from Table 3 with no identified real tidal disturbers, the strongest disturbers are found to be at the distances $600-2000 \mathrm{kpc}$, which leads to the long tail. BCGs with a merger morphology were excluded from the comparison with LSB galaxies.

Summarizing, we draw the following conclusion. BCGs/H II-galaxies significantly more often have the strongest disturbing neighbours at a distance of tens to a few hundred kpc than LSB dwarfs have. E.g., $\sim 76 \%$ of BCGs have such neighbours at $D_{\text {proj }}<400 \mathrm{kpc}$ while for LSB dwarfs this fraction is only $\sim 46 \%$. The median
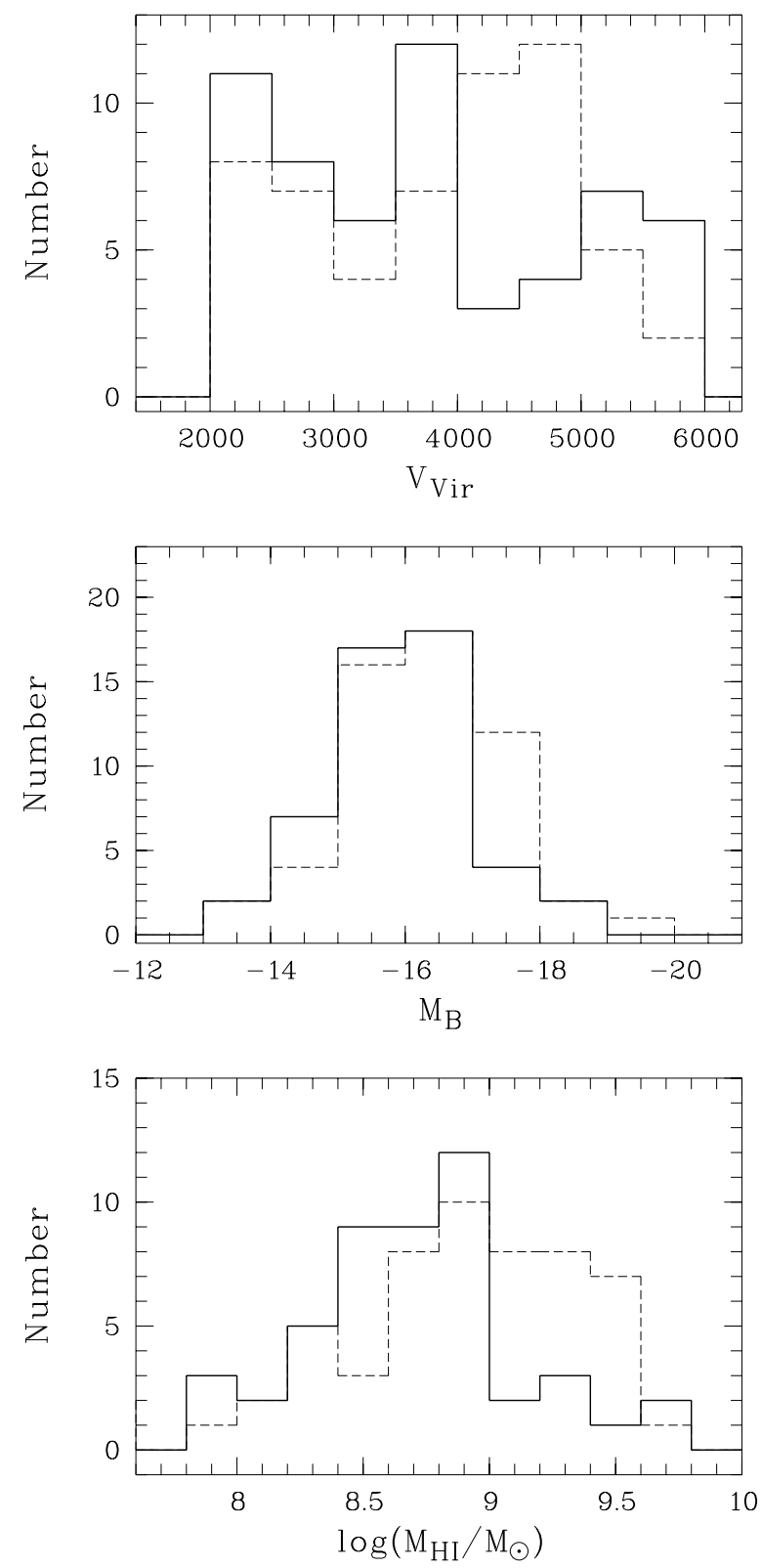

Fig. 3. Distributions of two compared samples - 49 BCGs from the general field (GF) region with $\mathrm{M}(\mathrm{HI})$ data (see 5.3) (solid line) and 56 LSB galaxies from Pildis et al. (1997) (dashed line), selected in the same velocity range. Upper panel: distribution of radial velocity; Middle panel: distribution of absolute $B$-band magnitude; Lower panel: distribution of $M(\mathrm{HI})$ (in solar masses).

and mean values of $D_{\text {proj }}-270$ and $384 \mathrm{kpc}$ for BCGs, and 560 and $728 \mathrm{kpc}$, respectively for LSB dwarfs, differ by about factor of two. In addition, $\sim 1 / 5$ of BCGs show merger morphology. If these 11 BCGs from the general field are included in the first bin $D_{\text {proj }}<100 \mathrm{kpc}$, the difference between BCGs and LSB dwarf distributions becomes striking.

Bothun et al. (1993) made a comparison of the local environment of a large LSB disk galaxy sample to that of 
HSB (high surface brightness) disks, and arrived a similar conclusion on the significant statistical deficit of galaxies from the CfA redshift survey within a projected radius of $0.5 \mathrm{Mpc}$ from LSB galaxies.

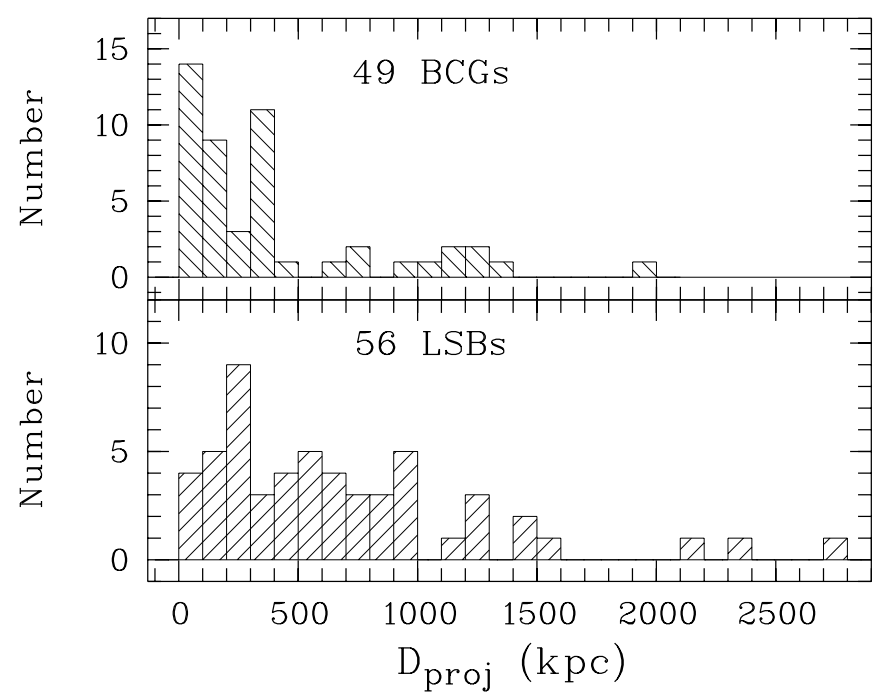

Fig. 4. Distribution of projected distances between the studied galaxies and their the most important disturbing neighbour, as found in NED. Upper panel: 49 BCGs from the general field group (see 5.3). Lower panel: 56 LSB galaxies from Pildis et al. (1997). Median values are $270 \mathrm{kpc}$ for BCGs and $560 \mathrm{kpc}$ for LSBDs.

Other than this exercise on brighter neighbouring galaxies, we can cite the results of the search for HIrich low-mass companions for the small LSB dwarf sample by Taylor (1997). It is shown that the control sample of LSB dwarfs has a significantly lower fraction of lowmass companions than the sample of BCG/H II-galaxies. However, several low-mass HI-companions do exist around LSB dwarfs, and they do not trigger SF bursts in these galaxies. This clearly indicates that the presence of a sufficiently close disturber cannot be the only factor determining enhanced SF in gas-rich galaxies. One plausible interpretation of this conclusion by Mihos et al. is mentioned in Sect. 5.5. On the other hand, $\sim 40 \%$ of their BCG sample have no detected companions. While the effect of incompletness probably affects this number, one can expect that some fraction of "isolated" BCGs are not triggered by interactions with low-mass companions.

Another sample, interesting for comparison, is the Local Volume sample (LV) (e.g., Karachentsev \& Makarov 1999), that is galaxies with radial velocities relative to the centroid of Local Group $V_{\mathrm{LG}}<$ $500 \mathrm{~km} \mathrm{~s}^{-1}$. Among 335 galaxies currently known in the LV (Makarov 2000), the class of BCG (or post Starburst) can be assigned to the following objects: NGC 1569 , NGC 1560, SBS 1123+576, Mkn 178, VII Zw 403, Arp 211, NGC 6789 . Of them SBS $1123+578$ is probably tidally disturbed and Mkn 178 is a merger, as shown in Table 3. The post Star-burst dwarf NGC 1569 interacts with
UGCA 92 at a distance of $\sim 40 \mathrm{kpc}$, or with a low-mass HI companion (Stil \& Israel 1998). The post Star-burst dwarf NGC 1560 is in the group Maffei/IC 342 at a distance of $\sim 300 \mathrm{kpc}$ from IC 342, which could disturb it, accounting for the time elapsed since beginning of the SF burst. For Arp 211, the situation is uncertain. Its photometrical distance of 2.8 Mpc (Nordic Optical Telescope - NOT data, Makarova et al. 1998), after obtained HST data changed to $6.7 \mathrm{Mpc}$ (Crone et al. 2001). Its probable strongest disturber, IC 3687, from the NOT photometry of 3 brightest blue stars is at about $3.0 \mathrm{Mpc}$. However, this estimate is probably not reliable, since from the estimate on the brightest red star, the distance is close to that of HST for Arp 211 (Makarova et al. 1998), and Arp 211 can be disturbed by IC 3687. The remaining BCGs, VII Zw 403 and NGC 6789 are probably well isolated. So, summarizing, we conclude that the data on a very limited number of star-bursting dwarfs in the LV do not contradict the results of the study on 86 BCGs above.

\subsection{Effect of global environment}

One of the important points of this study is to determine if the results obtained for the discussed BCG sample are representative of the general situation with BCGs. It is evident that some bias in the fraction of BCGs with the galaxy-interaction-induced SF burst must be present in our sample, since a significant number of our BCGs fall into the Local Supercluster, which is not typical of the general field. In paricular, besides the environments of the Virgo cluster, the galaxies of the UMa poor cluster (Tully et al. 1996) (with the center at RA $\sim 12^{\mathrm{h}}$, Dec. $\sim 49^{\circ}$, the angular diameter of $\sim 13^{\circ}$ and the radial velocity range $V_{\text {hel }}$ of 640 to $1150 \mathrm{~km} \mathrm{~s}^{-1}$ ) can appear among the BCGs of our sample.

The simplest way to check how much the effect of Local Supercluster contributes to the various types of interactions is to divide our sample by radial velocity. Roughly, the BCGs with the Virgo infall-corrected velocity $V_{\text {Vir }}<2000 \mathrm{~km} \mathrm{~s}^{-1}\left(D_{\mathrm{Vir}}<26.67 \mathrm{Mpc}\right)$ are considered constituents of the Local Supercluster volume, and all remaining as the galaxies of general field. The respective total numbers for these two groups are: 26 and 60 BCGs.

For the 26 BCGs within the "borders" of the Local Supercluster volume, we have the following statistics. 14 of them $(\sim 54 \pm 14 \%)$ have significantly brighter disturbing neighbours $(\Delta B \leq-1.5) .5$ BCGs $(\sim 19 \pm 8 \%)$ have neighbours of similar or lower luminosity, and $3(\sim 11.5 \pm 6.5 \%)$ have no neighbours but have a merger morphology. 4 more BCGs ( 15.5 $\pm 8 \%$ ) have neither evident companions (or have possible candidates with unknown velocities) nor show evidence of a recent merger.

For the remaining 60 BCGs in the general field, only 19 $(\sim 31.5 \pm 7 \%)$ have significantly brighter disturbing neighbours. 18 BCGs $(30 \pm 7 \%)$ have neighbours of similar or lower luminosity. 11 BCGs $(\sim 18.5 \pm 6 \%)$ have no disturbing neighbours, but have more or less clear indication of 
merger morphologies. The remaining 12 BCGs without evident companions or merging correspond to $20 \%$.

Comparing these two groups of BCGs from our sample, we see that the fraction of BCGs probably triggered by interactions with other galaxies is similar $(\sim 84.5 \pm 18 \%$ and $80 \pm 12 \%$, respectively) in the Local Supercluster volume and outside of it. The fractions of BCGs with various types of disturber and merger morphology differ in the two groups. However, only the difference in the fractions of BCGs with significantly brighter disturbing galaxies ( $\sim 54 \pm 14 \%$ versus $\sim 31.5 \pm 7 \%$ ) is seemingly real (while it is still within $1.5 \sigma$ of the combined uncertainty). This can quite naturally be interpreted as an indication of the more important role of interactions of BCGs with massive neighbours in the regions of higher galaxy density. The differences in the fractions of other categories (lowmass neighbours and mergers) are within the statistical uncertainties accounting for the small numbers of BCGs involved in the comparisons.

Thus, we conclude that in the general field, the role of galaxy interactions to trigger SF in BCGs is as important as in the Local Supercluster volume. $~ 80 \%$ of all BCGs are probably either tidally disturbed or result from recent mergers. At least $\sim 60 \%$ of field BCGs experience a tidal disturbance from other galaxies. Mergers and probable mergers constitute $\sim 1 / 5$ of all BCGs.

Some fraction of the remaining $\sim 20 \%$ of "noninteracting" BCGs can in fact also be interacting. Several of them have massive galaxies at projected distances of $\sim 500-600 \mathrm{kpc}$, and can be their distant companions. On the other hand, for several "non-interacting" BCGs, there are close candidate faint companions without radial velocities.

\subsection{On the results of complimentary approach}

Telles \& Maddox (2000) made an attempt to address the problem of faint companions of H II-galaxies through the 2 D statistical analysis of their environment using the APM galaxy catalog of the southern sky. They did not find an excess of faint galaxies around studied BCGs, and concluded that "tidal interactions cannot be the only factor that triggers their burst of star formation". We also do not generalize interactions as the only trigger of SF bursts in BCGs, but our results seem rather to contradict than to support each other. Therefore this requiress some discussion. Their conclusion is drawn from only one point on their cross-correlation function, corresponding to the minimal sampled distance of $120 h^{-1} \mathrm{kpc}$. This is about twice the typical distance between low-mass companions and BCGs in Taylor et al. (1995) and the median distance between BCGs and low-luminosity neighbours, in our sample (see Sect. 5.1). The presented uncertainty of this point is so high that it is difficult to trust any conclusion made on this basis. The auto-correlation function of the same H II-galaxies, determined by Iovino et al. (1988) and used by Telles \& Maddox (2000) as an argument, was measured only for distances larger than $350 h^{-1}$ kpc. Its extrapolation to the distances of interest, $\sim 50-80 h^{-1} \mathrm{kpc}$, is questionable in the frame of this problem, since its value is the subject of the study. To indicate possible caveats of their analysis, we note that about a half of our 32 BCGs with neighbours fainter than $M_{B}=-18^{\mathrm{m}} 5$ (see Table 3) are within the projected distances closer than $80 \mathrm{kpc}\left(60 h^{-1} \mathrm{kpc}\right) .3$ more such faint companions at larger distances are significantly fainter than $M_{B}=-15$, the limit for APM galaxies at the typical redshift of their H II-galaxies. Therefore, about $60 \%$ of faint companions found for SBS BCGs would probably not enter the crosscorrelation function of HII and APM galaxies, discussed by Telles \& Maddox.

\subsection{Trigger mechanism and BCG progenitors}

The question of the trigger mechanisms of BCG SF bursts has another interesting aspect relating to the evolutionary links of BCGs to other types of low-mass galaxies. What are BCGs in the periods between the episodes of enhanced SF activity? We do not know other gasrich low-mass galaxies without SF bursts other than dwarf spirals Sd-Sm, dwarf irregulars, including Im, and LSB dwarfs. Here we separate LSB dwarfs, as low-mass disks with roughly the same morphology classification, but with the central blue surface brightness $\mu_{0}(B) \geq$ $23 \mathrm{~m} 5 / \mathrm{sq}$ arcsec (e.g. Dalcanton et al. 1997), with the SF rates many times lower than their "normal" cousins. Unlike more typical Sdm and dIs, the HI surface density in LSBDs (almost) everywhere is below the threshold density of gravitational instability, resulting in global suppression of SF (van der Hulst et al. 1993; van Zee et al. 1997), and their dim optical appearance.

Evidently, either some or all types of gas-rich low-mass galaxies (in various proportions) can be progenitors of BCGs/H II-galaxies picked up by ELG surveys thanks to their strong emission lines. If, as we conclude, the main trigger mechanism of SF bursts in BCGs is external, then some consequences of BCG in relation to other galaxies can be derived.

As Mihos et al. (1997) showed in $N$-body simulations of collisions of equal mass high-surface (HSB) and lowsurface brightness (LSB) disk galaxies, their response during a close approach is qualitatively different. While in the HSB disk a bar structure was generated, which caused its gas to sink into the center, in the LSB disk only weak spirals were induced. Therefore even relatively strong tidal action of a neighbouring galaxy would cause only a small disturbance of the matter in a LSB galaxy. This leads to the conclusion that LSB dwarfs can hardly be progenitors of the main part of BCGs. Only the merger of LSB dwarfs with any type of low-mass galaxy will result in the strong disturbance and the loss of gas stability with subsequent collapse. In this case, their high HI mass content is the important factor in providing material for a strong SF burst. 
The merging of any type of gas-rich dwarf with an other dwarf will lead to a strong SF burst and an appearance of the BCG phenomenon. However, among the field BCGs of merger origin namely the fraction of LSB dwarf progenitors should prevail. This follows from the fact that the number density of LSB galaxies is several times higher than that of their HSB analogs (e.g., Dalcanton et al. 1997; O'Neil \& Bothun 2000). This should be the main factor determining the rate of merger BCGs, since, unlike the case of weak tidal trigger, the rate of merging is proportional to the square of the number density of galaxies under the question. Moreover, being the most abundant and stable type of galaxy in the general field, LSB dwarfs can constitute a significant, or even the main fraction of galaxies, exerting tidal action on BCG progenitors and triggering SF bursts in the majority of them.

Salzer \& Norton (1999) argued that BCG progenitors are not typical dwarf irregulars, but rather are objects from the extreme tail in their distribution on the central mass concentration. While these authors seemingly appreciate the important feature distinguishing the essential fraction of BCGs, their BCG sample seems to be randomly identified, and therefore the claimed results can be biased due to selection effects. The real fraction of this type of BCG should be confirmed on a BCG sample with well-defined selection criteria. If, however, Salzer \& Norton are right and such untypical dIrrs are the progenitors of the majority of observed BCGs, then such galaxies will be the most easily triggered by the tidal interactions from external galaxies. These authors suggest an intrinsic trigger mechanism, based on settling down of the cold ambient gas ejected from the disk during the previous SF burst. Unlike this, if interactions are the main trigger mechanism, the SF history, metal enrichment, gas consumption and the duty cycle of BCGs should noticebly depend on their environment. Some evidence for this come from the results of Izotov \& Guseva (1989) of the study of Virgo cluster BCGs. These have higher metallicities (mean $12+\log (\mathrm{O} / \mathrm{H}) \sim 8.3)$ than their typical general field analogs with a mean of $12+\log (\mathrm{O} / \mathrm{H}) \sim 8.0$ (e.g., Kniazev et al. 2001c). The systematic increase of a BCG parameter $M(\mathrm{HI}) / L_{B}$ with the decrease of galaxy density from a cluster through supercluster and general field to void environments (Pustilnik et al. 2001b) also supports the interaction-triggered SF bursts as the main mechanism.

New results of the blind HI survey for low-mass gasrich galaxies, presented by Schneider \& Rosenberg (2000), clearly demonstrate a steeply rising HI mass function of HI-rich field dwarf galaxies for masses $<10^{9} M_{\odot}$. This indicates once more the importance of low-mass companions of field dwarfs (often not seen without dedicated observations, e.g., Pisano \& Wilcots 1999) as probable triggers of SF activity. The hypothesis of infall of gas clouds from the intergalactic medium was recently discussed as an option for triggering SF bursts in "isolated" BCGs (e.g., Crone et al. 2001). By its essence such hypothesis is equivalent to the trigger due to sinking satellite if a gas-rich dwarf galaxy is considered as a massive component. Thus, this mechanism can in principle trigger SF bursts in some of BCGs of our sample without identified disturbing galaxy.

Summarizing the results of this study and the discussion above, we suggest that BCG progenitors are a mixture of various types of gas-rich low-mass galaxies with a wide range of surface densities and degrees of mass concentration. All of them, being isolated, are in a metastable state with various levels of "quasistationary, equilibrium" star formation rate. Some occasionally can "spontaneousely" leave it (i.e. due to some intrinsic processes) and undergo enhanced gas collapse, igniting SF burst. However, most reach the phase of SF burst by an external trigger consisting of various strength interactions with other galaxies. In particular, the disks with the highest central gas concentration are the least stable against gas collapse, and can be ingnited to SF burst by relatively weak tidals. On the other hand, LSB dwarfs are the most stable, and can be transformed to BCGs only by a merger with another galaxy.

Finally, we note some interesting implications of the described results for cosmological evolution of gas-rich low-mass galaxies. If galaxy interactions are the main trigger of enhanced SF in a significant number of gas-rich galaxies during the modern epoch, then their role at earlier times becomes dominant due to the significant increase of galaxy density. This is consistent with much observational data on the morphology and environment of faint high- $z$ galaxies discovered with the HST (see, e.g., review by Ferguson et al. 2000). Therefore, for most low-mass gasrich galaxies with a sufficiently high surface mass density, such as normal dIs, galaxy interactions during at least several first Gyr in the life of the Universe seemingly were the main driver of their cosmological evolution.

The same is applicable to the problem of the existence of local truly young galaxies. Such objects, if they are not disks of too low surface density, could survive to the modern epoch as protogalaxies, only if they populated relatively low galaxy density regions. Therefore, the general field environment, or even voids, are the most suitable regions to search for these very rare objects.

\subsection{Conclusions}

Summarizing the results and discussion presented above we draw the following conclusions:

1. The fraction of BCGs in the well-defined sample of 86 galaxies which either have the interacting neighbours with strong enough tidal action or have merger morphology is $\sim 80 \%$. This conclusion remains valid for BCG populating both the Local Supercluster volume and general field;

2. Fractions of BCGs with significantly brighter disturbing galaxies ("non-isolated" BCGs) vary from $54 \pm 14 \%$ for the Local Supercluster volume to $\sim 31.5 \pm 7 \%$ in the general field. This is consistent with the expectation 
that large mass galaxies play a more important role in the regions of higher galaxy density;

3. Among the so called "isolated" BCGs (without bright neighbouring galaxy) in both the Local Supercluster volume and in the general field, $\sim 43 \pm 10 \%$ are disturbed by dwarf galaxies and $\sim 26 \pm 8 \%$ have a merger morphology;

4. The whole BCG dataset discussed in the paper implies that galaxy interactions with both massive and dwarfs neighbours and with the full range of the disturbance amplitude (from weak tidals to mergers), is very important, and probably the main mechanism that triggers SF bursts in BCG progenitors. These are the mixture of all known low-mass gas-rich galaxy types with proportions which still are unknown. Depending on the quiescent level of SF of various BCG progenitors, their stability against various interaction-induced disturbances and their type of environment, interactions may be the main driver of their cosmological evolution. Future high quality $N$-body simulations of gas collapse in gas-rich galaxies due to the tidal action of external galaxies are necessary in order to support more certain conclusions.

\section{Appendix A: Spectral observations and results}

\section{A.1. Observations and reduction}

All observations have been conducted with the SAO RAS $6 \mathrm{~m}$ telescope during the period 1996-2000. Besides candidate companion galaxies, three SBS BCGs were also observed. For one of them (SBS 1413+495) the redshift was unknown before, and for two others we tried to improve the accuracy of redshifts to make more confident our search for companions. This optical redshift for SBS $1413+495$ was later confirmed and its precision was improved using HI data by Thuan et al. (1999). Candidate neighbours for two additional BCGs not entering into the sample of 86 BCGs were also observed, and appeared to be real companions (SBS $0916+542$ and $1120+586$ ). Observational results on these two new galaxies are given as well in Table A.1.

Three set-ups were used for the observations:

1. The first one was based on the spectrograph SP-124 in the Nasmyth- 1 focus with a Photometrics $1 \mathrm{~K} \times 1 \mathrm{~K}$ CCD detector (PM1024) with $24 \times 24 \mu \mathrm{m}$ pixel size. We used the gratings either with 300 grooves $/ \mathrm{mm}$ or with 600 grooves $/ \mathrm{mm}$. A long slit of $1^{\prime \prime} .5-3 .{ }^{\prime \prime} 0 \times 40^{\prime \prime}$ was used. The scale along the slit was $0.4^{\prime \prime} /$ pixel or $0.5^{\prime \prime} /$ pixel. Various spectral set-ups were used with dispersions from 2.4 to $5.5 \AA$ /pixel and a wavelength coverage of 4500-7000 A. More details on this set-up observations are given, e.g., in Pustilnik et al. (1999);

2. The second set-up was based on the spectrograph SP-124 in Nasmyth-1 focus with a Russian ISD017A $1040 \times 1160 \mathrm{CCD}$ detector with $16 \times 16 \mu \mathrm{m}$ pixel size and quantum efficiency $\approx 50 \%$ near $\sim 6000 \AA$. This setup was used only during 1 run in summer 1998, and few spectra were obtained. The same gratings and long slit were used as for the previous set-up;

3. The most recent observations were conducted with the spectrograph LSS (Afanasiev et al. 1995) in the prime focus and PM1024 CCD (as at first set-up) as a detector. Most of the long-slit spectra $\left(1^{\prime \prime} \cdot 2-2^{\prime \prime} .0 \times 180^{\prime \prime}\right)$ were obtained with the grating of 325 grooves $/ \mathrm{mm}$, giving a dispersion of $4.6 \AA /$ pixel (the spectral resolution 12 $15 \AA(F W H M))$ and the spectral range of 3600-8000. . The scale along the slit was $0.39^{\prime \prime} /$ pixel.

All observations were followed by recording the reference spectrum of a He-Ne-Ar lamp. Bias, dark noise and flat field were obtained every night. Observations of the spectrophotometrical standards from Bohlin (1996) were used to derive the sensitivity curve of the overall system. All observations and data acquisition were conducted using the NICE software package by Kniazev \& Shergin (1995) in the MIDAS ${ }^{4}$ environment.

Since these observations were performed mainly as a back-up program, in most of the cases the conditions were not photometric. Our main goal was to get radial velocity of the studied candidates. Therefore we discuss only this parameter.

Reduction was done as follows. Cosmic ray hits removal was done in MIDAS. The standard procedures of debiasing, flatfielding, wavelength and flux calibration were done in IRAF $^{5}$. Standard routines IDENTIFY, REIDENTIFY, FITCOORD, TRANSFORM were used to do the wavelength calibration and the correction for distortion and tilt for each frame. Then the one-dimensional spectra were extracted from each frame using the APALL routine without weighting. To derive the instrumental response function, we fitted the observed spectral energy distribution of the standard stars with a high-order polynomial.

Final measurements of the line intensities and positions and radial velocities were done in MIDAS. To improve the accuracy of the redshift determination, and further, to reduce possible small systematic shifts in the zero point of the wavelength calibration, we additionally checked the wavelengths of the night sky emission lines on the $2 \mathrm{D}$ spectra at the position of the object spectrum.

\section{A.2. Results of spectroscopy}

The objects observed are listed in Table A.1 containing the following information:

Column 1: The object's IAU-type name.

Column 2: Right ascension (RA) for equinox B1950.

\footnotetext{
${ }^{4}$ MIDAS is an acronym for the European Southern Observatory package - Munich Image Data Analysis System.

${ }^{5}$ IRAF is distributed by National Optical Astronomical Observatories, which is operated by the Association of Universities for Research in Astronomy, Inc., under cooperative agreement with the National Science Foundation.
} 
Column 3: Declination for equinox B1950.

Column 4: Apparent B-magnitude from APM database (Irwin 1998) which was recalculated to standard CCD $B$-magnitude using the calibration suggested by Kniazev et al. (2001b). Its rms uncertainty is 0.45 over the magnitude range $B=14^{\mathrm{m}} \cdot 0$ to $18^{\mathrm{m}} \cdot 5$.

Column 5: Heliocentric velocity and its rms uncertainty in $\mathrm{km} \mathrm{s}^{-1}$.

Column 6: Absolute B-magnitude calculated from the apparent $B$ magnitude and the heliocentric velocities. No correction for galactic extinction is made because all observed objects are located at high galactic latitudes and because the corrections are significantly smaller than the uncertainties of the magnitudes.

Column 7: Preliminary spectral classification type according to the presented spectral data. BCG/HII means that the galaxy possesses a characteristic HiI-region spectrum and a low enough luminosity $\left(M_{B} \geq-20\right)$. SBN are spiral galaxies of lower excitation with the central SF burst and the corresponding position in the line ratio diagrams, as discussed, e.g., in Ugryumov et al. (1999). Seyfert galaxies are separated mainly on the diagnostic diagrams as AGN. The criterion of broad lines was also used for the Sy classification. The ELG type means that an object has emission lines but is difficult to be classified using the existing data. ABS means a galaxy with the detected and identified absorption lines.

Column 8: One or more alternative names according to the information from NED.

Column 9: The list of spectral lines, well detected in the object spectrum and used for classification and/or redshift measurement.

All observed spectra are shown in Figs. A.1-A.3.

Acknowledgements. We are pleased to thank T. Kniazeva for the help in the reduction of observational data and D. Makarov for consultations. Our thanks to K. Noeske for sending us his and co-authors' article prior publication, and to J. Schombert, who kindly provided us with some unpublished data on the LSB galaxy sample. The authors thank the anonymous referee for useful suggestions. We acknowledge the partial support from Russian state program "Astronomy" and Center of Cosmoparticle Physics "Cosmion". This research has made use of the NASA/IPAC Extragalactic Database (NED) which is operated by the Jet Propulsion Laboratory, California Institute of Technology, under contract with the National Aeronautics and Space Administration. The use of the Digitized Sky Survey (DSS-II) and APM Database is gratefully acknowledged.

\section{References}

Afanasiev, V. L., Burenkov, A. N., Vlasyuk, V. V., \& Drabek, S. V. 1995, SAO RAS Internal Report, No. 234

Barnes, J. E., \& Hernquist, L. 1992, ARA\&A, 30, 705

Bernloehr, K. 1993, A\&A, 268, 25

Bohlin, R. C. 1996, AJ, 111, 1743

Bothun, G. D., Schombert, J. M., Impey, C. D., Sprayberry, D., \& McGaugh, S. S. 1993, AJ, 106, 530

Brinks, E. in Dynamics and Interactions of Galaxies, ed. R. Wielen (Springer-Verlag, Heidelberg), 146

Campos-Aguilar, A., \& Moles, M. 1991, A\&A, 241, 358
Carrasco, L., Tovmassian, H. M., Stepanian, J. M., et al. 1998, AJ, 115,1717

Chengalur, J. N., Giovanelli, R., \& Haynes, M. P. 1995, AJ, 109, 2415

Crone, M. M., Schulte-Ladbeck, R. E., Hopp, U., \& Greggio, L. 2001, ApJ, 545, L31

Dalcanton, J. J., Spergel, D. N., Gunn, J. E., Smith, M., \& Schneider, D. P. 1997, AJ, 114, 635

Doublier, V., Comte, G., Petrosian, A., Surace, C., \& Turatto, M. 1997, A\&AS, 124, 405

Falco, E. E., Kurtz, M. J., Geller, M. J., et al. 1999, PASP, 111, 438

Ferguson, H. C., Dickinson, M., \& Williams R. 2000, ARA\&A, 38,667

Georgiev, T., Karachentsev, I. D., \& Tikhonov, N. A. 1997, Astron. Lett., 23, 514

Gerola, H., Seiden, P. E., \& Schulman, L. S. 1980, ApJ, 242, 517

Hernquist, L., \& Minos, J. C. 1995, ApJ, 448, 41

Hunter, D. A. 1997, PASP, 109, 937

Icke, V. 1985, A\&A, 144, 115

Impey, C., \& Bothun, G. 1997, ARA\&A, 35, 267

Iovino, A., Melnick, J., \& Shaver, P. 1988, ApJ, 330, L17

Irwin, M. 1998, http://www.ast.cam.ac.uk/ ${ }^{\sim}$ apmcat/

Izotov, Y. I., \& Guseva, N. G. 1989, Afz, 30, 564

Izotov, Y. I., Lipovetsky, V. A., Guseva, N. G., Kniazev, A. Y., \& Stepanian, J. A. 1993, in DAEC Workshop The Feedback of Chemical Evolution on the Stellar Content of Galaxies, ed. D. Alloin, \& G. Stasinska, Meudon Obs., 217

Izotov, Y. I., Thuan, T. X., \& Lipovetsky, V. A. 1994, ApJ, 435,647

Izotov, Y. I., \& Thuan, T. X. 1999, ApJ, 511, 639

Joseph, R. D., Meikle, W. P. S., Robertson, N. A., \& Write, J. S. 1984, MNRAS, 209, 111

Karachentsev, I. D., \& Makarov, D. I. 1999, in Proc. IAU Symp. 186, Galaxy Interactions at Low and High Redshifts, ed. J. E. Barnes, \& D. B. Sanders, 109

Karachentseva, V. E., Prugniel, P., Vennik, J., et al. 1996, A\&AS, 117, 343

Keel, W. 1993, AJ, 106, 1771

Keel, W. C., \& Wu, W. 1995, AJ, 110, 129

Kennicutt, R. 1989, ApJ, 344, 685

Kniazev, A. Y., \& Shergin, V. S. 1995, SAO RAS Internal Report, No. 249, 1

Kniazev, A., Pustilnik, S., Masegosa, J., et al. 2000a, A\&A, 357,101

Kniazev, A. Y., Pramsky, A. G., Lipovetsky, V. A., et al. 2001a, in preparation

Kniazev, A. Y., Pustilnik, S. A., Ugryumov, A. V., et al. 2001b, in preparation

Kniazev, A. Y., Pustilnik, S. A., Ugryumov, A. V., Pramsky, A. G., \& Engels, D. 2001c, in Proc. of Euroconference Galaxy Evolution. I. Observational Clues, Granada, May 2000

Kraan-Korteweg, R. C. 1986, A\&AS, 66, 255

Kunth, D., \& Östlin, G. 2000, A\&AR, 10, 1

Larson, R. B., \& Tinsley, B. M. 1978, ApJ, 219, 46

Leitherer, C., Schaerer, D., Goldader, J. D., et al. 1999, ApJS, 123,3

Lipovetsky, V. A., et al. 2001, in preparation

Makarova, L., Karachentsev, I., Takalo, L., Heinamaki, P., \& Valtonen, M. 1998, A\&AS, 128, 459

Makarov, D. I. 2000, Ph.D. Thesis, SAO, Nizhnij Arkhyz 
Mazzarella, J. M., Bothun, G., \& Boroson, T. 1991, AJ, 101, 2034

Melnick, J. 1987, in Starburst and Galaxy Evolution, ed. T. X. Thuan, T. Montmerle, \& J. Tran Than Van (Éditions Frontières, Gif-sur-Yvette, France), 215

Mihos, J. C., \& Hernquist, L. 1994, ApJ, 425, L13

Mihos, J. C., McGaugh, S. S., \& de Blok, W. J. G. 1997, ApJ, 477, L79

Noeske, K. G., Iglesias-Páramo, J., Vilchez, J. M., Papaderos, P., \& Fricke, K. J. 2001, A\&A, 371, 806

Noguchi, M. 1988, A\&A, 203, 259

Olson, K. M., \& Kwan, J. 1990, ApJ, 349, 480; ApJ, 361, 426

O’Neil, K., \& Bothun, G. 2000, ApJ, 529, 811

Östlin, G., Amram, P., Bergvall, N., Masegosa, J., \& Boulesteix, J. 1999, A\&AS, 147, 419

Papaderos, P., Loose, H.-H., Fricke, K. J., \& Thuan, T. X. 1996, A\&A, 314, 59

Pildis, R. A., Schombert, J. M., \& Eder, J. A. 1997, ApJ, 481, 157

Pisano, D. J., \& Wilcots, E. M. 1999, AJ, 117, 2168

Pustilnik, S. A., Ugryumov, A. V., Lipovetsky, V. A., Thuan, T. X., \& Guseva, N. G. 1995, ApJ, 443, 499

Pustilnik, S. A., Engels, D., Ugryumov, A. V., et al. 1999, A\&AS, 137, 299

Pustilnik, S. A., Brinks, E., Thuan, T. X., Lipovetsky, V. A., \& Izotov, Y. I. 2001a, AJ, 121, 1413

Pustilnik, S. A., Martin, J.-M., Brosch, N. et al. 2001b, A\&A, submitted

Reshetnikov, V., \& Combes, F. 1997, A\&A, 324, 80

Rudnick, G., Rix, H.-W. \& Kennicutt, R. 2000, ApJ, 538, 569

Salzer, J. J. 1989, ApJ, 347, 152

Salzer, J. J., \& Norton, S. A. 1999, in Proc. of IAU Colloq. Low Surface Brightness Universe, ASP Conf. Ser., 170, ed. J. I. Davies, C. Impey, \& S. Phillipps, 253

Schneider, S., \& Rosenberg, J. 2000, in Mapping the Hidden Universe: The Universe Behind the Milky Way - The Universe in HI, ASP Conf. Ser., ed. R. C. KraanKorteweg, P. A. Henning, \& H. Andernach, in press [astro-ph/0010375]
Stil, J. M., \& Israel, F. P. 1998, A\&A, 337, 64

Sung, E.-C. 2000, PASP, 112, 121 (Disseration Summary)

Taylor, C. L., Brinks, E., \& Skillman, E. D. 1993, AJ, 105, 128

Taylor, C. L., Brinks, E., Pogge, R. W., \& Skillman, E. D. 1994, AJ, 107, 971

Taylor, C. L., Brinks, E., Grashuis, R. M., \& Skillman, E. D. 1995, ApJS, 99, 427

Taylor, C. L. 1997, ApJ, 480, 524

Telles, E., \& Maddox, S. 2000, MNRAS, 311, 307

Thuan, T. X. 1991, in Massive stars in Starbursts, ed. C. Leitherer, N. R. Walborn, T. M. Heckman, \& C. A. Norman (Cambridge, Cambridge Univ. Press), 183

Thuan, T. X. 1983, ApJ, 268, 667

Thuan, T. X., Izotov, Y. I., Lipovetsky, V. A., \& Pustilnik, S. A. in Proc. of ESO/OHP workshop Dwarf Galaxies, 1994, ed. G. Meilan, \& P. Prugniel, 421

Thuan, T. X., Izotov, Y. I., \& Lipovetsky, V. A. 1995, ApJ, 445,108

Thuan, T. X., Izotov, Y. I., \& Lipovetsky, V. A. 1996, ApJ, 463,120

Thuan, T. X., Lipovetsky, V. A., Martin, J.-M., \& Pustilnik, S. A. 1999, A\&AS, 139, 1

Toomre, A. 1964, ApJ, 139, 1217

Tully, R. B., Verheijen, M. A. W., Pierce, M. J., Huang, J.-S., \& Wainscoat, R. J. 1996, AJ, 112, 2471

van der Hulst, J. M., Skillman, E. D., Smith, T. R., et al. 1993, AJ, 106, 548

Ugryumov, A. V., Engels, D., Lipovetsky, V. A., et al. 1999, A\&AS, 135, 511

van Zee, L., Haynes, M. P., \& Salzer, J. J. 1997, AJ, 114, 2497 van Zee, L., Skillman, E. D., \& Salzer, J. J. 1998, AJ, 116, 1186 Vilchez, J. 1993, AJ, 110, 1090

Zaritsky, D., \& White, S. 1994, ApJ, 435, 599

Zaritsky, D., Smith, R., Frenk C., \& White S. D. M. 1997, ApJ, 478, 39 and 53

Zenina, O. A., Balinskaya, I. S., Kniazev, A. Y., \& Lipovetsky, V. A. 1997, Astron. Rep., 41, 472 